\title{
Iceberg-type Problems: Estimating Hidden Parts of a Continuum from the Visible Parts
}

\author{
Roger W. Barnard *, Kent Pearce ${ }^{* *}$, and Alexander Yu. Solynin *** $\dagger$ \\ Department of Mathematics and Statistics, Texas Tech University, Box 41042, Lubbock, TX 79409
}

Received 21 March 2007, revised 30 May 2008, accepted 16 June 2008

Published online XXXX

Key words omitted area problem, logarithmic capacity, univalent function, symmetrization, local variation MSC (2000) $30 \mathrm{C} 70$

\begin{abstract}
We consider the complex plane $\mathbb{C}$ as a space filled by two different media, separated by the real axis $\mathbb{R}$. We define $\mathbb{H}_{+}=\{z: \Im z>0\}$ to be the upper half-plane. For a planar body $E$ in $\mathbb{C}$, we discuss a problem of estimating characteristics of the "invisible" part, $E_{-}=E \backslash \mathbb{H}_{+}$, from characteristics of the whole body $E$ and its "visible" part, $E_{+}=E \cap \mathbb{H}_{+}$. In this paper, we find the maximal draft of $E$ as a function of the logarithmic capacity of $E$ and the area of $E_{+}$.
\end{abstract}

Copyright line will be provided by the publisher

\section{Introduction}

We will discuss problems (called iceberg-type problems below) of estimating characteristics of the "invisible" part of a compact set $E$ in the complex plane $\mathbb{C}$ from some known characteristics of the whole set and its "visible" part. We emphasize from the beginning that the problems we study in this paper are not directly related to (real) physical icebergs. The problem name reflects the fact that the object under consideration consists of two parts, hidden and visible, and the question is to recover some of the properties of the hidden part from the visible part. During the ages a titanic work has been done to solve this problem in its everyday physical setting.

In this paper, we study iceberg-type problems in two-dimensional space, which will be identified as the complex plane $\mathbb{C}$. Accordingly, $\overline{\mathbb{C}}=\mathbb{C} \cup\{\infty\}, \mathbb{H}_{+}=\{z: \Im z>0\}$, and $\mathbb{H}_{-}=\{z: \Im z<0\}$ will denote the extended complex plane, the upper half-plane, and the lower half-plane, respectively. The real axis $\mathbb{R}$ will play the role of the surface of interface between $\mathbb{H}_{+}$and $\mathbb{H}_{-}$.

For any given compact set $E$ in $\mathbb{C}$, we define $E_{+}=E \cap \mathbb{H}_{+}$and $E_{-}=E \cap \overline{\mathbb{H}}_{-}$. The sets $E_{+}$and $E_{-}$denote the visible and hidden parts of $E$, respectively.

An accumulative characteristic of any body $E$ surrounded by media is its potential or capacity. In our twodimensional setting, the logarithmic capacity will be chosen as the primary characteristic of $E$. We remind the reader that the logarithmic capacity, cap $E$, of a compact set $E$ is defined by

$$
-\log \operatorname{cap} E=\lim _{z \rightarrow \infty}(g(z)-\log |z|),
$$

where $g(z)$ denotes Green's function of the unbounded component $D(E)$ of $\overline{\mathbb{C}} \backslash E$ having singularity at $z=\infty$. Let $\mathcal{F}$ be the collection of all continua (= connected compact sets) $E$ in $\mathbb{C}$ such that

$$
\operatorname{cap}(E)=1 \text {. }
$$

\footnotetext{
* E-mail: roger.w.barnard@ttu.edu, Phone: 806742 2566, Fax: 8067421112

** Corresponding author: E-mail: kent.pearce@ttu.edu, Phone: 806742 2566, Fax: 8067421112

*** E-mail: alex.solynin@ttu.edu, Phone: 806742 2566, Fax: 8067421112

$\dagger$ Partially supported by NSF grant DMS-0525339
} 


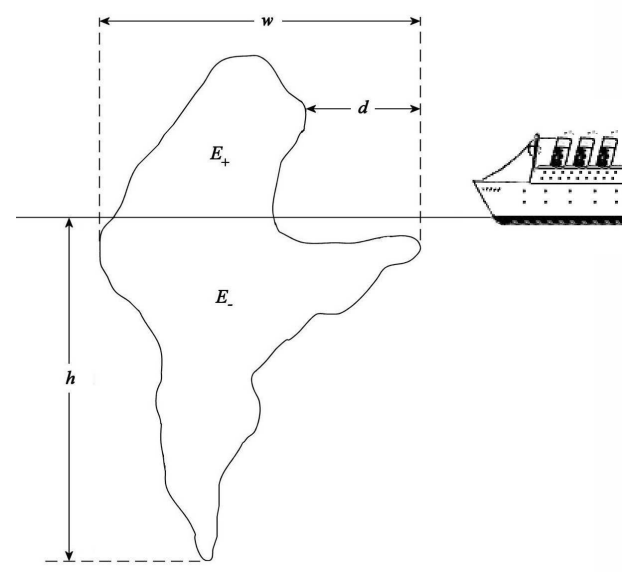

Fig. 1 Two-dimensional iceberg.

For the measured characteristic of a visible part $E_{+}$we will choose the mass of $E_{+}$, which, assuming homogeneity of $E$, is proportional to the area of $E_{+}$. For $E$ in $\mathcal{F}$, the well known estimates of the logarithmic capacity show that

$$
0 \leq \operatorname{area}\left(E_{+}\right) \leq \text {area }(E) \leq \pi(\operatorname{cap}(E))^{2}=\pi
$$

Characteristics of the hidden part $E_{-}$which one may want to control and which are of a particular importance, include: the draft of the iceberg $H(E)$, the width of the invisible part of the iceberg $w(E)$, and the safe distance from the iceberg $d(E)$. Figure 1 illustrates these characteristics while the precise definitions are as follows:

$$
H(E)=\max (-\Im(z))
$$

where the maximum is taken over all $z$ in $E$,

$$
w(E)=\max \left(\Re\left(z_{2}-z_{1}\right)\right),
$$

where the maximum is taken over all $z_{1}, z_{2}$ in $E_{-}$, and

$$
d(E)=\max \left(\Re\left(z_{2}\right)\right)-\sup \left(\Re\left(z_{1}\right)\right),
$$

where the maximum is taken over all $z_{2}$ in $E_{-}$and the supremum is taken over all $z_{1}$ in $E_{+}$.

Then, the extremal problem for each of the functionals (1.1), (1.2), and (1.3) is to find its maximal value over the class $\mathcal{F}$ and describe all possible extremal continua. We define
(a) $H(\mathcal{F})=\max H(E)$
(b) $w(\mathcal{F})=\max w(E)$
(c) $d(\mathcal{F})=\max d(E)$,

where in each case the maximum is taken over all sets $E$ in $\mathcal{F}$.

Our main goal in this paper is to give a complete solution to problem (1.4)(a). Problems (1.4)(b) and (1.4)(c) along with some other questions will be discussed in the last section.

As is well known, problems on the logarithmic capacity of simply-connected continua can be reformulated as problems about functions in the class $\Sigma^{\prime}$ of univalent functions

$$
f(z)=z^{-1}+a_{0}+a_{1} z+\cdots,
$$


which are analytic in the unit disk $\mathbb{D}$, except for a simple pole at $z=0$. For $f$ in $\Sigma^{\prime}$, define $E_{f}=\overline{\mathbb{C}} \backslash f(\mathbb{D})$ and define $\Sigma_{0}^{\prime}=\left\{f \in \Sigma^{\prime}: 0 \in E_{f}\right\}$.

We will solve problem (1.4)(a) by solving its reformulated dual problem for the class $\Sigma_{0}^{\prime}$. There is a technical advantage in shifting to the dual problem in that the analytical and constructional difficulties which surround the dual problem are more tractable than those in the original setting. The precise formulation of the dual of the maximal draft problem (1.4)(a) is the following problem on the maximal omitted area for the class $\Sigma_{0}^{\prime}$. For any given real $h$ such that $0<h<4$, find

$$
A(h):=\max \text { area }\left(E_{f} \cap\{w: \Re w>h\}\right),
$$

where the maximum is taken over all $f$ in $\Sigma_{0}^{\prime}$, and find all functions $f$ in $\Sigma_{0}^{\prime}$ extremal for (1.6). Thus, the question is, for any given $h$, such that $0<h<4$, to maximize the area omitted by the functions $f$ in $\Sigma_{0}^{\prime}$ in the half-plane $\mathbb{H}_{h}:=\{w: \Re w>h\}$.

We note here that our parameter $h$, which is equal to the horizontal distance from $w=0$ to the half-plane $\mathbb{H}_{h}$, gives as well the value of the maximal draft of icebergs with visible area $A=A(h)$. In addition, in Corollary 1.2 (below) we show that the extremal configuration for problem (1.4)(a) coincides with the extremal configuration for problem (1.6) up to rotation and translation.

For convenience we define $A_{f}(h)=$ area $\left(E_{f} \cap \mathbb{H}_{h}\right)$. The maximal omitted area problem (1.6) is solved by the following theorem.

Theorem 1.1 Let $h$ satisfy $0<h<4$ and let $f$ belong to $\Sigma_{0}^{\prime}$. Then,

$$
A_{f}(h) \leq \pi \beta^{2}-2 \beta h r\left(1-r^{2}\right) \int_{\tau}^{1}\left(\frac{t\left(1-t^{2}\right) \sqrt{1-\tau^{2} t^{2}}}{\left(r^{2}+t^{2}\right)^{2} \sqrt{t^{2}-\tau^{2}}}-\frac{\left(1-t^{2}\right) \sqrt{t^{2}-\tau^{2}}}{t\left(1+r^{2} t^{2}\right)^{2} \sqrt{1-\tau^{2} t^{2}}}\right) d t
$$

where $r=r(h)$ is the solution to the equation

$$
h=2 \beta r\left(1-r^{2}\right) \int_{0}^{\tau} \frac{t\left(1-t^{2}\right) \sqrt{1-\tau^{2} t^{2}}}{\left(r^{2}+t^{2}\right)^{2} \sqrt{\tau^{2}-t^{2}}} d t
$$

which is unique for $0<r<1$ and where

$$
\tau=\sqrt{\frac{\left(1+r^{2}\right) \sqrt{2-2 r^{2}+r^{4}}-\left(1+r^{4}\right)}{1+3 r^{2}}}
$$

and

$$
\beta=\frac{4 r \sqrt{\left.r^{2}+\tau^{2}\right)}}{\left(1+r^{2}\right)^{2} \sqrt{\left.1+\tau^{2} r^{2}\right)}}
$$

Equality occurs in (1.7) if and only if $f=f_{h}$ with $f_{h}(z)=F\left(\psi_{r}^{-1}(z)\right)$ with $r$ defined by (1.8), where

$$
z=\psi_{r}(s)=\frac{\left(1-r^{2}\right) s-r\left(1-s^{2}\right)}{\left(1-r^{2}\right) s+r\left(1-s^{2}\right)}, \quad s \in \mathbb{D}_{+}:=\{s \in \mathbb{D}: \Re s>0\},
$$

maps the semidisk $\mathbb{D}_{+}$conformally onto the unit disk $\mathbb{D}$ and

$$
F(s)=-2 \beta r\left(1-r^{2}\right) \int_{0}^{s} \frac{t\left(t^{2}+1\right) \sqrt{1+\tau^{2} t^{2}}}{\left(t^{2}-r^{2}\right)^{2} \sqrt{t^{2}+\tau^{2}}} d t
$$

with the principal branches of the radicals and with $\tau$ and $\beta$ defined by (1.9) and (1.10). 


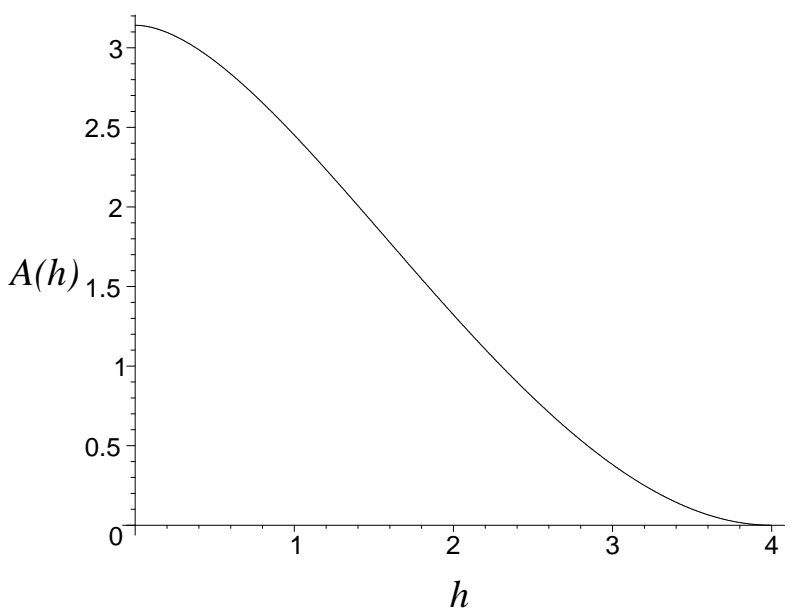

Fig. 2 Maximal omitted area $A(h)$.

Theorem 1.1 shows that the maximal omitted area $A(h)$ is given by the explicit expression in the right-hand side of (1.7) with $r, \tau$, and $\beta$ defined by (1.8), (1.9), and (1.10), respectively. Its graph shown in Figure 2 suggests, and we will prove this in Lemma 2.1 in Section 2, that $A(h)$ strictly decreases from $\pi$ to 0 as $h$ runs from 0 to 4 . Therefore, the inverse, $h=\Psi(A)$, of the function $A(h)$ is well defined on $0 \leq A \leq \pi$.

Corollary 1.2 If $E \in \mathcal{F}$ has the visible area $A, 0<A<\pi$, i.e. if area $\left(E_{+}\right)=A$, then the draft of $E$ is restricted by

$$
H(E) \leq \Psi(A)
$$

where the function $\Psi$ is defined above.

Equality occurs in (1.13) if and only if E coincides with the continuum $i\left(E_{f_{h}}-h\right)$, where $h=\Psi(A)$ and $f_{h}$ is given in Theorem 1.1, up to a horizontal drift.

The extremal shapes $E(h)=E_{f_{h}}$ for some typical values of $h$ are displayed in Figure 3 . As in previous works on this subject (see [1]- [9]) the boundary $\partial E(h)$ consists of the so-called free boundary $L_{f r}$ that is an open Jordan arc in $\mathbb{H}_{h}$ having its ends at the points $h \pm i a$ for some $a$, where $0<a<4$ and the non-free boundary $L_{n f}$, which consists of a horizontal segment $[0, h]$ and two vertical segments $[h, h+i a]$ and $[h, h-i a]$, see Figure 3 . The precise definitions will be postponed until Section 2 .

The function $z=\psi(s)$ defined by (1.11) with $0<r<1$ maps the semidisk $\mathbb{D}_{+}$conformally onto $\mathbb{D}$ such that $\psi(r)=0$. This reveals the role of the parameter $r$. The parameters $\tau$ and $\beta$ defined by (1.9) and (1.10) also have special meanings. Namely, the function $F(s)$ maps the segments $[-i,-i \tau]$ and $[i \tau, i]$ onto the vertical segments of the boundary of the corresponding extremal configuration, see Figure 3. In addition, we will prove in Section 2 for an extremal function $f_{h}$ that $\left|f_{h}^{\prime}\left(e^{i \theta}\right)\right|=\beta$ for all $e^{i \theta}$ in the free $\operatorname{arc} l_{f r}=f_{h}^{-1}\left(L_{f r}\right)$.

To prove Theorem 1.1, we apply techniques developed in [9], [6], and [7]. These techniques use symmetrizationtype transformations to prove some a priori smoothness of the boundary, which in turn allows us to apply Juliatype local variations to find boundary values of the extremal function. To show that the extremal function can be recovered from its boundary values and is unique for every $h$, we prove in Section 4 several monotonicity lemmas. In each case, we use a Sturm sequence argument as an essential tool in our proofs.

We want to mention two other alternative methods, which may work in the omitted area problems studied in this paper. The first method is based on the Alt-Caffarelli variational technique which was developed by J. Lewis in 
[16]. His approach does not require any a priori smoothness and has been found to be very efficient for omitted area problems, see [16] and [4]. The second approach, which was applied in [2] and [8], uses Steiner symmetrization to reduce the problem to the class of typically-real functions. Then, the well-known integral representation for this class could be used to characterize the extremal functions.

\section{Extremal configurations and functions}

In this section, we collect preliminary results about existence and geometric properties of extremal functions and configurations. For notational convenience, we define $\mathbb{D}_{r}(w)=\{z:|z-w|<r\}$, with $\mathbb{D}_{r}=\mathbb{D}_{r}(0)$, and $l_{x}=\{z: \Re z=x\}$; that is, $\mathbb{D}_{r}(w)$ is the disk centered at $w$ of radius $r$ and $l_{x}$ is the vertical line through the point $x$ on the real axis.

Lemma 2.1 (a) For every $h$, where $0 \leq h \leq 4$, there exists $f$ in $\Sigma_{0}^{\prime}$ such that $A_{f}(h)=A(h)$. In addition, $A(h)$ is continuous and strictly decreasing in $0 \leq h \leq 4$.

(b) If $f$ is extremal for $A(h)$, then $E_{f}=\mathbb{C} \backslash f(\mathbb{D})$ possesses Steiner symmetry with respect to $\mathbb{R}$ and circular symmetry with respect to the ray $\mathbb{R}_{0}:=\{z: \Re z \geq 0\}$.

(c) For $0<h<4$, the boundary $\partial E_{f}$ consists of a free boundary $L_{f r}$ and non-free boundary $L_{n f}$. The nonfree boundary $L_{n f}$ consists of a horizontal segment $I(h)=[0, h]$ and two vertical segments (possibly degenerate) $v_{f}^{+}=\left[h, h+i a_{f}\right]$ and $v_{f}^{-}=\left[h, h-i a_{f}\right]$ with some $0 \leq a_{f}<4$ depending on $f$.

The free boundary $L_{f r}$ is an open Jordan rectifiable arc in $\mathbb{H}_{h}$ joining the points $h \pm i a_{f}$. In addition, $\hat{L}=L_{f r} \cup\left[-i a_{f}, i a_{f}\right]$ is a closed Jordan curve that satisfies the following Lavrent'ev condition:

$$
\operatorname{length}\left(J\left(w_{1}, w_{2}\right)\right) \leq C\left|w_{1}-w_{2}\right| \quad \text { for } w_{1}, w_{2} \text { in } \hat{L},
$$

where $C$ is a constant independent of $w_{1}, w_{2}$ and $J\left(w_{1}, w_{2}\right)$ denotes the shortest arc of $\hat{L}$ between $w_{1}$ and $w_{2}$.

Proof. (a) Since the omitted area functional $A_{f}(h)$ is upper semi-continuous, the existence of an extremal function, at least one for each $h$, follows from the compactness of the class $\Sigma_{0}^{\prime}$. Since $E_{f} \subset\{w:|w| \leq 4\}$ for all $f$ in $\Sigma_{0}^{\prime}$, a similar compactness argument easily implies the continuity of $A(h)$.

Since for any given $f$ in $\Sigma_{0}^{\prime}$, the area $A_{f}(h)$ does not increase in $0 \leq h \leq 4$, the non-strict monotonicity of $A(h)$ is obvious. Let $0 \leq h_{1}<h_{2} \leq 4$. Then, it follows from the property of the free boundary in part (c), which is proved below, that if $f$ is extremal for $A\left(h_{2}\right)$, then $f$ can not be extremal for $A\left(h_{1}\right)$. Therefore, $A(h)$ is strictly decreasing on $0 \leq h \leq 4$.

(b) Symmetry properties can be established via a standard argument using appropriate Steiner and circular symmetrizations, cf. [9], [6], [7].

(c) Symmetry properties of the extremal configurations together with the subordination principle, see [14], imply the assertion about the non-free boundary $L_{n f}$.

To rule out the case that $L_{f r}$ consists of multiple arcs in $\mathbb{H}_{h}$ having their ends on the real axis, we apply polarization. For the definition and properties of this transformation the reader may consult [11], [18], [9].

Let $f$ in $\Sigma_{0}^{\prime}$ be an extremal for $A(h)$ and let $p=\max _{w \in E_{f}}\{\Re w\}$ and $p_{h}=(p+h) / 2$. For real $\tau$, let $E_{f, \tau}^{+}=E_{f} \cap \overline{\mathbb{H}}_{\tau}, E_{f, \tau}^{-}=E_{f} \backslash \mathbb{H}_{\tau}$, and let $E_{f, \tau}^{*}$ denote the set symmetric to $E_{f, \tau}^{+}$w.r.t. the vertical line $l_{\tau}=\{w: \Re w=\tau\}$. We claim that $E_{f}$ satisfies the following polarization property (cf. [9]):

$$
E_{f, \tau}^{*} \subset E_{f, \tau}^{-} \quad \text { for all } \tau \text { such that } p_{h} \leq \tau<p .
$$

Indeed, if $E_{f, \tau}^{*} \not \subset E_{f, \tau}^{-}$for some $\tau, p_{h} \leq \tau<h$, then $\widehat{E}_{f, \tau}^{p} \neq E_{f}$, where $\widehat{E}_{f, \tau}^{p}$ denotes the polarization of $E_{f}$ into the half-plane $\mathbb{H}_{h}^{-}$. It is also obvious that $\widehat{E}_{f, \tau}^{p}$ is not a reflection of $E_{f}$ in the line $l_{\tau}$. Then, the principle of polarization implies the following strict inequality

$$
\operatorname{cap}\left(\widehat{E}_{f, \tau}^{p}\right)<\operatorname{cap} E_{f}
$$

which easily leads to a contradiction to our assumption that $f$ is extremal for $A(h)$. 

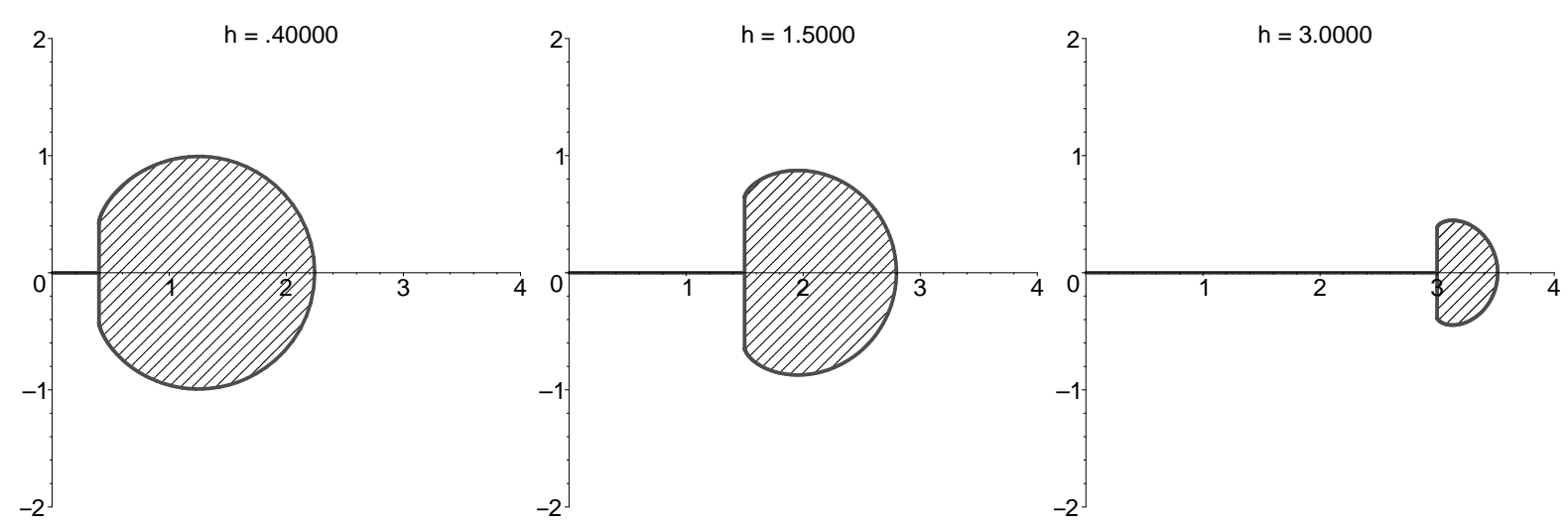

Fig. 3 Extremal shapes $E(h)$.

Now the symmetry properties of $E_{f}$ when combined with the polarization property (2.2) show that $L_{f r}$ is an open Jordan arc in $\mathbb{H}_{h}$ joining the points $h \pm i a_{f}$ with some $0 \leq a_{f}<4$ depending on $f$.

Next, since $E_{f}$ is bounded, Steiner symmetric w.r.t. $\mathbb{R}$, and circularly symmetric w.r.t. $\mathbb{R}_{0}$, the proof of Lemma 2.2 in [7] shows that $\hat{L}$ satisfies the Lavrent'ev condition (2.1). In particular, $\hat{L}$ and therefore $L_{f r}$ are rectifiable. The proof of the lemma is complete.

For $f$ in $\Sigma_{0}^{\prime}$ which is extremal for $A(h)$, we define $l_{f r}=\left\{e^{i \theta}:|\theta|<\theta_{1}\right\}$ be the "free arc"; that is, $l_{f r}$ is the preimage of $L_{f r}$ under the mapping $f$. Similarly, we define $l_{v}^{ \pm}=f^{-1}\left(v_{f}^{ \pm}\right)$and $l_{h}^{ \pm}=f^{-1}\left(I^{ \pm}(h)\right)$, where $I^{+}(h)$ and $I^{-}(h)$ denote, respectively, the upper part and the lower part of the segment $I(h)$. We also define $e^{ \pm i \theta_{1}}=f^{-1}\left(h \pm i a_{f}\right)$ and $e^{ \pm i \theta_{2}}=f^{-1}(h \pm i 0)$. Also, we define $l_{v}=l_{v}^{+} \cup l_{v}^{-}$and $l_{h}=l_{h}^{+} \cup l_{h}^{-}$.

Lemma 2.2 For a given $h, 0<h<4$, let $f$ in $\Sigma_{0}^{\prime}$ be extremal for $A(h)$. Then, there exists a positive $\beta$ such that

(a) For every sufficiently small positive $\varepsilon, f^{\prime}$ is bounded on the compact set $\overline{\mathbb{D}} \backslash\left\{\mathbb{D}_{\varepsilon} \cup \mathbb{D}_{\varepsilon}\left(e^{i \theta_{2}}\right) \cup \mathbb{D}_{\varepsilon}\left(e^{-i \theta_{2}}\right)\right\}$;

(b) $\left|f^{\prime}(z)\right|=\beta$ if $z \in l_{f r}$;

(c) $\left|f^{\prime}\left(e^{i \theta}\right)\right|$ strictly increases from $\beta$ to $\infty$ as $\theta$ runs from $\theta_{1}$ to $\theta_{2}$;

(d) The vertical non-free boundary is not degenerate, i.e. $a_{f}>0$;

(e) $\left|f^{\prime}(z)\right| \rightarrow \beta$ as $z \rightarrow e^{i \theta_{1}}$ such that $z \in \overline{\mathbb{D}}$.

Proof. (a) First we prove that $f^{\prime}$ is bounded near $l_{f r}$. If not then there is $e^{i \theta_{0}}$ in $l_{f r}$ and a sequence $z_{k} \rightarrow e^{i \theta_{0}}$ such that $z_{k} \in \mathbb{D}$ for all $k$ in $\mathbb{N}$ and $f^{\prime}\left(z_{k}\right) \rightarrow \infty$.

Let $\varphi_{k}$ denote the conformal mapping from $\mathbb{D}$ onto the domain $\mathbb{D} \backslash \overline{\mathbb{D}}_{\varepsilon_{k}}\left(z_{k}\right)$ with $\varepsilon_{k}=1-\left|z_{k}\right|$ normalized by $\varphi_{k}(0)=0, \varphi_{k}^{\prime}(0)>0$ and define $f_{k}=\beta_{k} f \circ \varphi_{k}$ with $\beta_{k}=1-\pi^{2} \varepsilon_{k}^{2} / 6$. One can easily verify (see, for example, Lemma 3.1 in [9]) that $f_{k} \in \Sigma_{0}^{\prime}$. Since $0<h<4$ and $\operatorname{diam}\left(E_{f_{k}}\right) \leq 4$ by the well-known Faber's inequality, an elementary geometric estimate gives

$$
\text { area }\left(E_{f_{k}} \backslash \mathbb{H}_{h}\right) \leq 4 \pi^{2} \varepsilon_{k}^{2} .
$$

Using (2.3) and the mean value property of the subharmonic function $\left|f^{\prime}(z)\right|^{2}$, we can estimate the area $A_{f_{k}}(h)$ as follows:

$$
\begin{aligned}
A_{f_{k}}(h) & =\quad \beta_{k}^{2}\left(A_{f}(h)+\operatorname{area}\left(f\left(\mathbb{D}_{z_{k}}\left(\varepsilon_{k}\right)\right)\right)\right)-\operatorname{area}\left(E_{f_{k}} \backslash \mathbb{H}_{h}\right) \\
& \geq \beta_{k}^{2}\left(A_{f}(h)+\pi \varepsilon_{k}^{2}\left|f^{\prime}\left(z_{k}\right)\right|^{2}\right)-4 \pi^{2} \varepsilon_{k}^{2} \geq A_{f}(h)+\left(\pi\left|f^{\prime}\left(z_{k}\right)\right|^{2}-C\right) \varepsilon_{k}^{2}+o\left(\varepsilon_{k}^{2}\right),
\end{aligned}
$$


with some constant $C>0$ independent of $f$. Since $f^{\prime}\left(z_{k}\right) \rightarrow \infty$ as $k \rightarrow \infty$, (2.4) contradicts the extremality of $f$. Therefore, $f^{\prime}$ is bounded near $l_{f r}$.

(b) First we show that $\left|f^{\prime}(z)\right|$ is constant a.e. on $l_{f r}$. Since $L_{f r}$ is Jordan locally rectifiable, it follows that the non-zero finite limit

$$
f^{\prime}(\zeta)=\lim _{z \rightarrow \zeta, z \in \overline{\mathbb{D}}} \frac{f(z)-f(\zeta)}{z-\zeta} \neq 0, \infty
$$

exists a.e. on $l_{f r}$; see [17, Theorem 6.8, Exercise 6.4.5]. Assume that

$$
0<\beta_{1}=\left|f^{\prime}\left(e^{i \nu_{1}}\right)\right|<\left|f^{\prime}\left(e^{i \nu_{2}}\right)\right|=\beta_{2}<\infty
$$

for $e^{i \nu_{1}}, e^{i \nu_{2}} \in l_{f r}$. Note that (2.5) and (2.6), combined with the fact that $f^{\prime}$ is bounded near $l_{f r}$, allow us to apply the two-point variational formulas, see [9, Lemma 10] or [6, Lemma 5]. Namely, for fixed positive $k_{1}, k_{2}$ such that $0<k_{1}<1<k_{2}$ and $k_{1} \beta_{1}^{-1}>k_{2} \beta_{2}^{-1}$ and fixed $\varphi>0$ small enough, we consider the two-point variation $\tilde{D}$ of $D=f(\mathbb{D})$ centered at $w_{1}=f\left(e^{i \nu_{1}}\right)$ and $w_{2}=f\left(e^{i \nu_{2}}\right)$ with inclinations $\varphi$ and radii $\varepsilon_{1}=k_{1} \varepsilon, \varepsilon_{2}=k_{2} \varepsilon$, respectively; see [6, Section 2]. Computing the change in the area by [6, formula (2.11)], we find

$$
\text { Area }(\mathbb{C} \backslash \tilde{D})-\text { Area } E_{f}=\frac{2 \pi \varphi-\sin 2 \pi \varphi}{2 \sin ^{2} \pi \varphi} \varepsilon^{2}\left(k_{1}^{2}-k_{2}^{2}\right)+o\left(\varepsilon^{2}\right)>0
$$

for all $\varepsilon>0$ small enough. Similarly, applying [6, formula (2.10)], we get

$$
\frac{\operatorname{cap}(\mathbb{C} \backslash \tilde{D})}{\operatorname{cap}\left(E_{f}\right)}=\left[\frac{\varphi(2+\varphi)}{6(1+\varphi)^{2}} \frac{k_{1}^{2}}{\beta_{1}^{2}}-\frac{\varphi(2-\varphi)}{6(1-\varphi)^{2}} \frac{k_{2}^{2}}{\beta_{2}^{2}}\right] \varepsilon^{2}+o\left(\varepsilon^{2}\right)<0
$$

for all $\varepsilon>0$ small enough and $\varphi$ chosen such that the expression in the brackets is positive.

Inequalities (2.7) and (2.8) lead to a contradiction to the extremality of $f$ for $A(h)$, via a standard subordination argument. Thus $\left|f^{\prime}\left(e^{i \theta}\right)\right|=\beta$ a.e. on $l_{f r}$ with some $\beta>0$.

To prove that $\left|f^{\prime}\left(e^{i \theta}\right)\right|=\beta$ everywhere on $l_{f r}$, we consider the auxiliary conformal mapping

$$
g=\varphi \circ f \circ k_{\tau} \quad \text { with } \varphi(w)=1 /\left(w-p_{h}\right),
$$

where $p_{h}$ is defined in the proof of Lemma 2.1, and with

$$
k_{\tau}(\zeta)=k^{-1}(\tau k(\zeta)), \quad \text { where } k(\zeta)=\zeta /(1-\zeta)^{2} \text { and } \tau=1 / \sin ^{2}\left(\theta_{2} / 2\right) .
$$

We note that $k_{\tau}$ maps the slit disk $\mathbb{D}^{\prime}=\mathbb{D} \backslash\left[-1,-r_{0}\right]$, where $r_{0}=(\sqrt{\tau}-\sqrt{\tau-1})^{2}$, conformally and one-to-one onto $\mathbb{D}$ in such a way that the radial slit is mapped onto the $\operatorname{arc} l_{h}=\left\{e^{i \theta}:|\theta-\pi| \leq \pi-\theta_{2}\right\}$.

Let $D_{g}^{\prime}=g\left(\mathbb{D}^{\prime}\right)$ and let $D_{g}=D_{g}^{\prime} \cup\left(\left(p_{h}-h\right)^{-1},-p_{h}^{-1}\right]$. By the Schwarz reflection principle, the function $g$ can be continued to a function, still denoted by $g$, which maps the whole disk $\mathbb{D}$ conformally and one-to-one onto $D_{g}$. It follows from Lemma 2.1(c) that $D_{g}$ is a bounded Jordan domain, whose boundary satisfies the Lavrent'ev condition (2.1) for some $C>0$. Therefore, $D_{g}$ is a Smirnov domain; see [17, Sections 7.3, 7.4]. Thus, $\log \left|g^{\prime}\right|$ can be represented by the Poisson integral

$$
\log \left|\varphi^{\prime}(w) f^{\prime}(z) k_{\tau}^{\prime}(\zeta)\right|=\log \left|g^{\prime}(\zeta)\right|=\frac{1}{2 \pi} \int_{0}^{2 \pi} P(r, \psi-t) \log \left|g^{\prime}\left(e^{i t}\right)\right| d t
$$

with boundary values defined a.e. on $\mathbb{T}$; see [17, p. 155]. Equation (2.10) easily implies that

$$
\left|g^{\prime}\left(e^{i \psi}\right)\right|=\beta \mid \varphi^{\prime}\left(f\left(k_{\tau}\left(e^{i \psi}\right)\right)|| k_{\tau}^{\prime}\left(e^{i \psi}\right) \mid\right.
$$

for all $e^{i \psi}$ such that $k_{\tau}\left(e^{i \psi}\right) \in l_{f r}$ and therefore $\left|f^{\prime}\left(e^{i \theta}\right)\right|=\beta$ for all $e^{i \theta} \in l_{f r}$. In addition, (2.10) implies that $\log f^{\prime}$ is bounded on $\overline{\mathbb{D}}$ outside any neighborhoods of the points $z=0, z=-1$, and $z=e^{ \pm i \theta_{2}}$. 
(c) Since $E_{f}$ is Steiner symmetric w.r.t. $\mathbb{R}$, the strict monotonicity of $\left|f^{\prime}\right|$ along $l_{v}^{+}$follows from [9, Lemma 4]. To prove that $\left|f^{\prime}\left(e^{i \theta}\right)\right|>\beta$ for all $e^{i \theta} \in l_{v} \backslash\{0\}$, we assume that $\beta=\left|f^{\prime}\left(e^{i \nu_{1}}\right)\right|>\left|f^{\prime}\left(e^{i \nu_{2}}\right)\right|=\beta_{2}$ with $e^{i \nu_{1}} \in l_{f r}$ and some $e^{i \nu_{2}} \in l_{v}^{+}$. Then, applying the two-point variation as above, we get inequalities (2.7) and (2.8), contradicting the extremality of $f$ for $A(h)$, again via a subordination argument. Hence, $\left|f^{\prime}\left(e^{i \theta}\right)\right| \geq \beta$ for all $e^{i \theta} \in l_{v}$ which, when combined with the strict monotonicity property of $\left|f^{\prime}\right|$, leads to the strict inequality $\left|f^{\prime}\left(e^{i \theta}\right)\right|>\beta$ for $e^{i \theta} \in l_{v}$.

(d) Assume that $a_{f}=0$. Then, $\theta_{1}=\theta_{2}, L_{n f}=I(h)$, and $\hat{L}=L_{f r} \cup\{h\}$. In addition, $\left|f^{\prime}\left(e^{i \theta}\right)\right|=\beta>0$ for all $e^{i \theta} \in l_{f r}$ by part (b) of this proof.

In the notation of part (b), we consider the function $g=\varphi \circ f \circ k_{\tau}$ defined by (2.9), which maps $\mathbb{D}$ conformally onto the domain $D_{g}$. As we have mentioned above, $\log \left|g^{\prime}(\zeta)\right|$ can be represented by the Poisson integral (2.10).

Since $\left|k_{\tau}^{\prime}\left(e^{i \psi}\right)\right| \rightarrow 0$ as $\psi \rightarrow \pi$, it follows that $\left|g^{\prime}\left(e^{i \psi}\right)\right|=\beta\left|\varphi^{\prime}\left(k_{\tau}\left(e^{i \psi}\right)\right)\right|\left|k_{\tau}^{\prime}\left(e^{i \psi}\right)\right| \rightarrow 0$ as $\psi \rightarrow \pi$. Therefore,

$$
\log \left|g^{\prime}\left(e^{i \psi}\right)\right| \rightarrow-\infty \quad \text { as } \psi \rightarrow \pi .
$$

From (2.10) and (2.11), using the well-known properties of the radial limits of the Poisson integral, we obtain that

$$
\log \left|g^{\prime}(-r)\right| \rightarrow-\infty \quad \text { as } r \rightarrow 1^{-}
$$

Now we show that $g$ has a finite non-zero angular derivative at $\zeta=-1$. To do this, we construct two comparison functions $f_{1}$ and $f_{2}$. Let $f_{1}$ map $\mathbb{D}$ conformally onto the vertical strip $\{w: 0<\Re w<h\}$ such that $f_{1}(0)=h / 2$, $f_{1}(-1)=h$ and let $g_{1}=\varphi \circ f_{1}$. Then, of course, $g_{1}^{\prime}(-1)$ exists and $g_{1}^{\prime}(-1) \neq 0, \infty$. Since $g_{1}(\mathbb{D}) \subset g(\mathbb{D})$ and $g_{1}(-1)=g(-1)=1 /\left(h-p_{h}\right)$, we can apply the comparison Theorem 4.14 in [17] to conclude that $g$ has the angular derivative $g^{\prime}(-1)$ and

$$
\left|g^{\prime}(-1)\right|=c_{1}\left|g_{1}^{\prime}(-1)\right| \quad \text { where } 0 \leq c_{1}<\infty \text {. }
$$

Next we construct our second comparison function. We define $K_{p_{h}}=E_{f} \cap \overline{\mathbb{H}}_{p_{h}}$ and $K_{p_{h}}^{*}$ be the set symmetric to $K_{p_{h}}$ w.r.t. the vertical line $l_{p_{h}}$. Define $\Omega=\mathbb{C} \backslash\left(K_{p_{h}} \cup K_{p_{h}}^{*}\right)$, let $f_{2}$ map $\mathbb{D}$ conformally onto $\Omega$ such that $f_{2}(0)=\infty, f_{2}(-1)=h$, and let $g_{2}=\varphi \circ f_{2}$. Since the boundary $\partial \Omega$ is analytic in a vicinity of $w=h$, it follows that $g_{2}^{\prime}(-1)$ exists and $g_{2}^{\prime}(-1) \neq 0, \infty$.

It follows from equation $(2.2)$ in the proof of Lemma 2.1(c) that $g(\mathbb{D}) \subset g_{2}(\mathbb{D})$. Now, Theorem 4.14 in [17] implies that

$$
\left|g_{2}^{\prime}(-1)\right|=c_{2}\left|g^{\prime}(-1)\right| \quad \text { where } 0 \leq c_{2}<\infty .
$$

This together with (2.13) shows that the finite non-zero angular derivative $g^{\prime}(-1)$ exists. Now Proposition 4.7 [17] implies that $g^{\prime}(\zeta)$ has the finite angular limit $g^{\prime}(-1)$ at $\zeta=-1$ where $g^{\prime}(-1) \not 0$. In particular,

$$
\left|g^{\prime}(-r)\right| \rightarrow\left|g^{\prime}(-1)\right| \neq 0 \quad \text { as } r \rightarrow 1^{-}
$$

contradicting (2.12). This proves that $a_{f}>0$.

(e) To show that $\left|f^{\prime}\right|$ is continuous at $e^{ \pm i \theta_{1}}$, we again use the function $g$ defined by (2.9). Using Theorem 4.14 in [17] with $g_{1}$ defined in part (d) of this proof as a comparison function, we conclude that the finite angular derivative $g^{\prime}\left(k_{\tau}^{-1}\left(e^{i \theta_{1}}\right)\right)$, and therefore the angular derivative $f^{\prime}\left(e^{i \theta_{1}}\right)$, exists finitely.

By the reflection principle, $f$ can be continued analytically across $l_{v}^{-}$. By Lemma $2.1, E_{f}$ is Steiner symmetric w.r.t. $\mathbb{R}$ and circularly symmetric w.r.t. $\mathbb{R}_{0}$. Using these facts it is not difficult to see that this analytic continuation, say $\tilde{f}$, of $f$ is univalent in the disk $U=\left\{z:\left|z-\varepsilon_{0} e^{\left.i\left(\theta_{1}+\theta_{2}\right) / 2\right)}\right|<\rho_{0}\right\}$ for a sufficiently small positive $\varepsilon_{0}$ and $\rho_{0}=\left|e^{i \theta_{1} / 2}-\varepsilon_{0} e^{i \theta_{2} / 2}\right|$. By Proposition 4.9 [17], the function $\tilde{f}$ has the angular derivative $\tilde{f}^{\prime}\left(e^{i \theta_{1}}\right)$ at $z=e^{i \theta_{1}}$, which of course coincides with the angular derivative $f^{\prime}\left(e^{i \theta_{1}}\right)$.

We have $l_{v}^{-} \subset U$. Since $\left|f^{\prime}\left(e^{i \theta}\right)\right|$ is monotone and greater than $\beta$ on $l_{v}^{-}$, it follows that $\lim _{\theta \rightarrow \theta_{1}^{+}} f^{\prime}\left(e^{i \theta}\right)=\beta_{0} e^{-i \theta_{1}}$ where $0<\beta \leq \beta_{0}$. Therefore,

$$
f^{\prime}(z) \rightarrow \beta_{0} e^{-i \theta_{1}} \quad \text { as } \quad z \rightarrow e^{i \theta_{1}}
$$


in any Stolz angle in $\mathbb{D}$ with the vertex at $e^{i \theta_{1}}$. To show that $\beta_{0}=\beta$, we use the Poisson integral (2.10). Let $\psi_{1}=\arg \left(k_{\tau}^{-1}\left(e^{i \theta_{1}}\right)\right)$. If $\beta_{0} \neq \beta$, then the theorem about radial limits of the Poisson integral implies that

$$
\lim _{r \rightarrow 1^{-}} \log \left|g^{\prime}\left(r e^{i \psi_{1}}\right)\right|=\frac{1}{2} \lim _{\varepsilon \rightarrow 0} \log \left|g^{\prime}\left(e^{i\left(\psi_{1}+\varepsilon\right)}\right) g^{\prime}\left(e^{i\left(\psi_{1}-\varepsilon\right)}\right)\right| .
$$

This implies that $\left|f^{\prime}\left(k_{\tau}\left(r e^{i \theta_{1}}\right)\right)\right| \rightarrow \sqrt{\beta \beta_{0}}$ as $r \rightarrow 1^{-}$, which together with (2.14) shows that we must have $\beta_{0}=\beta$.

Using the Poisson integral (2.10) once more, we conclude that $\log \left|g^{\prime}(\zeta)\right|$ is continuous for $\zeta$ such that $|\zeta| \leq 1$ and $\left|\zeta-k_{\tau}^{-1}\left(e^{i \theta_{1}}\right)\right|$ is small enough. Since $g=\varphi \circ f \circ k_{\tau}$ and $\varphi$ and $k_{\tau}$ are conformal in the corresponding domains the latter implies (e).

The proof of Lemma 2.2 is complete.

\section{Closed form of the extremal functions and the proof of Theorem 1.1}

Lemmas 2.1 and 2.2 provide sufficient information to find a closed form of the function $f$ extremal for $A(h)$ when $0<h<4$. It is convenient to work in the auxiliary $s$-plane with $z=\psi_{r}(s)$ defined by (1.11). We note that this auxiliary mapping was already used in [2] to solve the minimal area $a_{2}$-problem for convex functions.

The function $z=\psi_{r}(s)$ maps the semidisk $\mathbb{D}_{+}$conformally onto $\mathbb{D}$ such that

$$
\psi_{r}(r)=0, \quad \psi_{r}(i)=e^{i \theta(r)},
$$

where

$$
\theta(r)=2 \arcsin \frac{2 r}{1+r^{2}}
$$

Lemma 3.1 Let $f$ be extremal for $A(h), 0<h<4$, and let $F_{r}(s)=f\left(\psi_{r}(s)\right), 0<r<1$. Then, there are parameters $r, \tau, \beta$ where $0<r<1,0<\tau<1$, and $\beta>0$ such that

$$
F_{r}^{\prime}(s)=-\frac{2 \beta r\left(1-r^{2}\right) s\left(s^{2}+1\right)\left(1+\tau^{2} s^{2}\right)^{1 / 2}}{\left(s^{2}-r^{2}\right)^{2}\left(s^{2}+\tau^{2}\right)^{1 / 2}}
$$

with the principal branches of the radicals.

Proof. Let $\theta_{1}$ and $\theta_{2}$ be the angles defined for $f$ as in Section 2. Since $\theta(r)$ defined by (3.1) strictly increases in $0<r<1$, its inverse, $r(\theta)$, is well defined. Choose $r=r\left(\theta_{1}\right)$. For this $r$, let $i \tau=\psi_{r}^{-1}\left(e^{i \theta_{2}}\right)$. Then, $0<\tau<1$. By Lemma 2.2, there is a positive $\beta$ such that $\left|f^{\prime}\left(e^{i \theta}\right)\right|=\beta$ for all $|\theta| \leq \theta_{1}$.

Let $\Phi(s)=\Phi(s ; r, \tau, \beta)$ denote the expression in the right-hand side of (3.2) considered as a function of $s \in \mathbb{D}_{+}$ for the values of $r, \tau$, and $\beta$ chosen above.

It follows from (1.5) and (1.11) that the limit

$$
\lim _{s \rightarrow r}\left(F_{r}^{\prime}(s) / \Phi(s)\right)=\frac{4 r\left(r^{2}+\tau^{2}\right)^{1 / 2}}{\beta\left(1+r^{2}\right)^{2}\left(1+r^{2} \tau^{2}\right)^{1 / 2}}
$$

exists and is finite and non-zero. Using (3.3) one can easily show that the function

$$
g(s)=u(s)+i v(s):=\log \left(F_{r}^{\prime}(s) / \Phi(s)\right)
$$

is analytic and single-valued on $\mathbb{D}_{+}$.

It follows from Lemma 2.1 and the definition of $\Phi(s)$ that $g(s)$ takes real values on the vertical diameter $[-i, i]$ except its three singularities at the points $s=0, s=i \tau$, and $s=-i \tau$. By the Schwarz reflection principle, $g(s)$ can be continued as an analytic multi-valued function in the punctured disk $\mathbb{D}^{\prime}=\mathbb{D} \backslash\{0, \pm i \tau\}$. 
To analyze the nature of the multi-valuedness of $g$, we compute the periods $\omega_{0}, \omega_{1}$, and $\omega_{-1}$ of $g$ at the singularities $s=0, s=i \tau$, and $s=-i \tau$, respectively.

Since $F_{r}(s)$ maps the segments $[-i \tau, 0]$ and $[0, i \tau]$, each one-to-one onto the horizontal segment $[0, h]$, it follows that $F_{r}(s)$ is analytic near $s=0$ and its Taylor expansion at $s=0$ has the form $F_{r}(s)=C s^{2}+\cdots$ where $C \neq 0$. Then, we have

$$
\frac{F_{r}^{\prime \prime}(s)}{F_{r}^{\prime}(s)}=\frac{1}{s}+\text { non-negative powers of } s .
$$

Using this, we easily find that

$$
\omega_{0}=\int_{|s|=\varepsilon} d g(s)=\int_{|s|=\varepsilon}\left(\frac{F_{r}^{\prime \prime}(s)}{F_{r}^{\prime}(s)}-\frac{\Phi^{\prime}(s)}{\Phi(s)}\right) d s=0
$$

for all sufficiently small positive $\varepsilon$. Similarly, we find

$$
\omega_{1}=\int_{|s-i \tau|=\varepsilon} d g(s)=\int_{|s-i \tau|=\varepsilon}\left(\frac{F_{r}^{\prime \prime}(s)}{F_{r}^{\prime}(s)}-\frac{\Phi^{\prime}(s)}{\Phi(s)}\right) d s=0 .
$$

By symmetry, we also have $\omega_{-1}=0$.

Since all periods of $g$ are zero, the function $g(s)$ is analytic and single-valued on $\mathbb{D}$.

We claim that $u(s):=\Re g(s) \equiv 0$ on $\mathbb{D}$. To prove this, we test the boundary values of $u$. For $s=e^{i t}$ with $|t|<\pi / 2$, using Lemma 2.2(b) and (1.11) we compute

$$
\left|F_{r}^{\prime}(s)\right|=2 \beta r\left(1-r^{2}\right) \frac{\left|s^{2}+1\right|}{\left|s^{2}-r^{2}\right|^{2}}=|\Phi(s)|,
$$

which shows that $u\left(e^{i t}\right)=0$ for $|t|<\pi / 2$. By Lemma 2.2(e), $\left|f^{\prime}(z)\right| \rightarrow \beta$ as $z \rightarrow e^{i \theta_{1}}$. Using the explicit expressions for $\psi_{r}$ and $\Phi$, see (1.11) and (3.2), we easily find that $\left|F_{r}^{\prime}(s) / \Phi(s)\right| \rightarrow 1$ as $s \rightarrow i$. Thus, $u$ has boundary value 0 at $s=i$. By symmetry, $u\left(e^{i t}\right)=0$ everywhere on $\mathbb{T}$.

Since $u$ is harmonic in $\mathbb{D}$ and continuous on $\overline{\mathbb{D}}$, the maximum principle implies that $u(s) \equiv 0$ on $\mathbb{D}$. Then, of course, $g(s)$ is constant on $\mathbb{D}$, and this constant has the value 0 since $\Im g(r)=0$ by (3.3). This proves the lemma.

Using the closed form (3.2) combined with some computational results, the proofs of which are postponed until Section 4 , we can prove our main theorem.

Proof of Theorem 1.1. If $0<h<4$, let $f$ be an extremal function for $A(h)$, which exists by Lemma 2.1. Let $F_{r}(s)=f\left(\psi_{r}(s)\right)$ be defined as in Lemma 3.1. Then, $F_{r}^{\prime}(s)$ has the form (3.2).

We claim that there is a unique set of parameters $r=r(h), \tau=\tau(h)$, and $\beta=\beta(h)$, for which the function $f_{h}(z)=F_{r}\left(\psi_{r}^{-1}(z)\right)$, with $F_{r}(s)$ defined by (3.2), is in $\Sigma_{0}^{\prime}$. Then, of course, $f_{h}$ will be the unique extremal for $A(h)$.

Expanding (3.2) into a Laurent series at $s=r$, we obtain

$$
F_{r}^{\prime}(s)=\frac{A_{-2}}{(s-r)^{2}}+\frac{A_{-1}}{s-r}+A_{0}+\text { positive powers of }(s-r),
$$

where

$$
A_{-2}=-\frac{\beta\left(1-r^{4}\right)\left(1+r^{2} \tau^{2}\right)^{1 / 2}}{2\left(r^{2}+\tau^{2}\right)^{1 / 2}}
$$

and

$$
A_{-1}=-\frac{\beta r\left(1-r^{2}\right)\left(\left(1+3 r^{2}\right) \tau^{4}+2\left(1+r^{4}\right) \tau^{2}-\left(1-r^{2}\right)\right)}{2\left(r^{2}+\tau^{2}\right)^{3 / 2}\left(1+r^{2} \tau^{2}\right)^{1 / 2}}
$$


Since $F_{r}(s)$ is a single-valued function in $\mathbb{D}_{+}$, we must have $A_{-1}=0$. This gives

$$
\tau=\sqrt{\frac{\left(1+r^{2}\right) \sqrt{2-2 r^{2}+r^{4}}-\left(1+r^{4}\right)}{1+3 r^{2}}},
$$

which is equation (1.9) of Theorem 1.1.

To find $\beta$, we use the normalization $\lim _{z \rightarrow 0}\left(-z^{2} f^{\prime}(z)\right)=1$. Then, using (1.11) and (3.4), we obtain

$$
1=\lim _{s \rightarrow r}\left(-\psi_{r}^{2}(s) \frac{F^{\prime}(s)}{\psi_{r}^{\prime}(s)}\right)=\lim _{s \rightarrow r}\left(\frac{\beta(s-r)^{2}\left(1+r^{2}\right)^{2}\left(1+\tau^{2} s^{2}\right)^{1 / 2}}{4 r^{2}(s-r)^{2}\left(s^{2}+\tau^{2}\right)^{1 / 2}}\right) .
$$

From this we find

$$
\beta=\frac{4 r\left(r^{2}+\tau^{2}\right)^{1 / 2}}{\left(1+r^{2}\right)^{2}\left(1+r^{2} \tau^{2}\right)^{1 / 2}},
$$

which is equation (1.10) of Theorem 1.1.

Next, using conditions $f\left(e^{i \theta_{2}}\right)=F_{r}(i \tau)=h$, we can find an equation that links $r$ and $h$ :

$$
h=\int_{0}^{i \tau} F^{\prime}(s) d s=2 \beta r\left(1-r^{2}\right) \int_{0}^{\tau} \frac{t\left(1-t^{2}\right)\left(1-\tau^{2} t^{2}\right)^{1 / 2}}{\left(t^{2}+r^{2}\right)^{2}\left(\tau^{2}-t^{2}\right)^{1 / 2}} d t,
$$

which is equation (1.8) of Theorem 1.1 .

Let $h(r)$ denote the right-hand side of (1.8) with $\tau$ and $\beta$ considered as functions of $r$ defined by (1.9) and (1.10). In Lemma 4.2 in Section 4, we will show that $h(r)$ strictly decreases from 4 to 0 as $r$ runs from 0 to 1. Therefore, for every $h$, such that $0<h<4$, (1.8) has a unique solution $r=r(h)$ whenever $0<r<1$.

Thus, we have proven that for every $0<h<4$, there is a unique function $f_{h}$ in $\Sigma_{0}^{\prime}$ extremal for $A(h)$. In addition, we have shown that the derivative $F_{r}^{\prime}(s)=f_{h}^{\prime}\left(\psi_{r}(s)\right) \psi_{r}^{\prime}(s)$, where $r=r(h)$ is defined by (1.8), is given by (3.2). Integrating (3.2), we obtain (1.12).

To complete the proof of Theorem 1.1, we have to find the maximal omitted area $A(h)$. This calculation will be given in Lemma 4.1 below.

\section{Area functional and monotonicity lemmas}

Lemma 4.1 For $0<h<4$, the maximal omitted area $A(h)$ is given by

$$
A(h)=\pi \beta^{2}-2 \beta h r\left(1-r^{2}\right) \int_{\tau}^{1}\left(\frac{t\left(1-t^{2}\right) \sqrt{1-\tau^{2} t^{2}}}{\left(r^{2}+t^{2}\right)^{2} \sqrt{t^{2}-\tau^{2}}}-\frac{\left(1-t^{2}\right) \sqrt{t^{2}-\tau^{2}}}{t\left(1+r^{2} t^{2}\right)^{2} \sqrt{1-\tau^{2} t^{2}}}\right) d t
$$

with $r, \tau$, and $\beta$ defined in Theorem 1.1.

Proof. Let $f$ be extremal for $A(h)$ and let $F_{r}(s)$ with $r=r(h)$ be defined for $f$ as in Lemma 3.1. Applying the standard line integral formula for the area, we find

$$
A(h)=\frac{1}{2} \Im \int_{\partial E_{f}} \bar{w} d w=\frac{1}{2} \Im \int_{h+a i}^{h-a i} \bar{w} d w+\frac{1}{2} \Im \int_{L_{f r}} \bar{w} d w=-h a+\frac{1}{2} \Im \int_{L_{f r}} \bar{w} d w,
$$

where

$$
a=\Im f\left(e^{i \theta_{1}}\right)=\Im \int_{i \tau}^{i} F_{r}^{\prime}(s) d s=\beta r\left(1-r^{2}\right) \int_{\tau}^{1} \frac{2 t\left(1-t^{2}\right) \sqrt{1-\tau^{2} t^{2}}}{\left(t^{2}+r^{2}\right)^{2} \sqrt{t^{2}-\tau^{2}}} d t .
$$



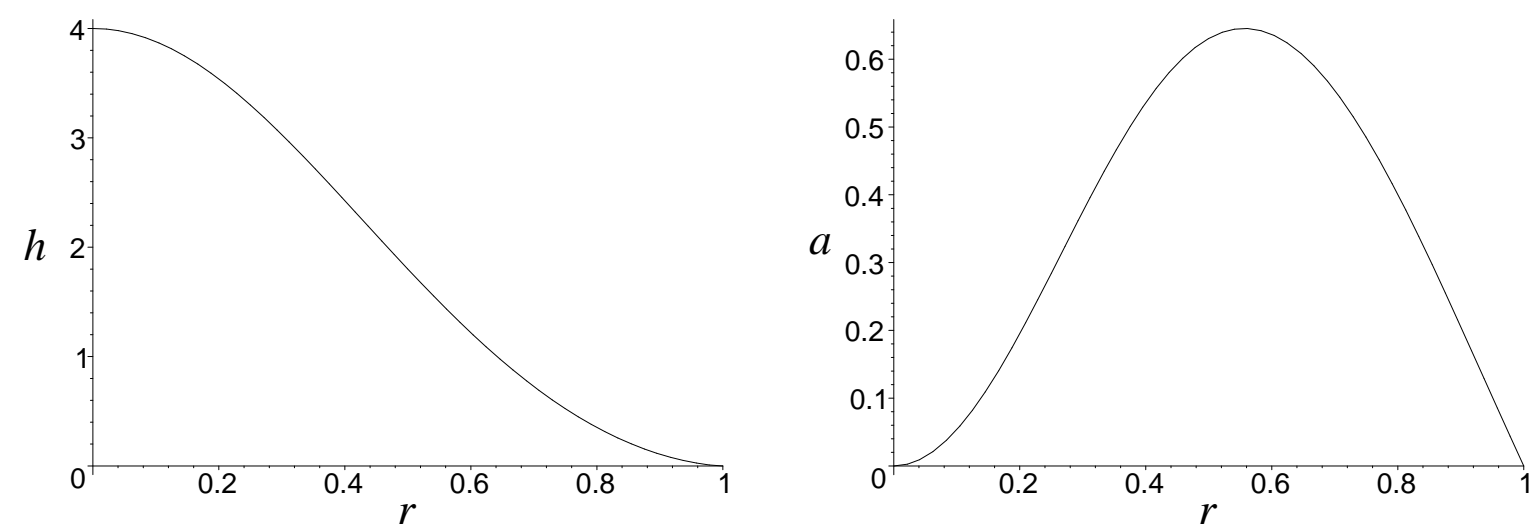

Fig. 4 Functions $h(r)$ and $a(r)$.

Now, taking the condition $\left|f^{\prime}(z)\right|=\beta$ for $z \in l_{f r}$ into account, we find the integral over the free boundary:

$$
\begin{aligned}
\frac{1}{2} \Im \int_{L_{f r}} \bar{w} d w & =\frac{1}{2} \Re \int_{\theta_{1}}^{-\theta_{1}} f\left(e^{i \theta}\right) e^{-i \theta} \overline{f^{\prime}\left(e^{i \theta}\right)} d \theta=\frac{\beta^{2}}{2} \Re \int_{\theta_{1}}^{-\theta_{1}} \frac{f\left(e^{i \theta}\right) e^{i \theta}}{e^{2 i \theta} f^{\prime}\left(e^{i \theta}\right)} d \theta \\
& =-\frac{\beta^{2}}{2} \Im \int_{\mathbb{T}} \frac{f(z)}{z^{2} f^{\prime}(z)} d z+\frac{\beta^{2}}{2} \Re \int_{\theta_{1}}^{2 \pi-\theta_{1}} \frac{f\left(e^{i \theta}\right)}{e^{i \theta} f^{\prime}\left(e^{i \theta}\right)} d \theta \\
& =-\frac{\beta^{2}}{2} \Im \operatorname{Res}\left[\frac{f(z)}{z^{2} f^{\prime}(z)}, 0\right]+\beta^{2} h \int_{\theta_{1}}^{\theta_{2}} \frac{d \theta}{\left|f^{\prime}\left(e^{i \theta}\right)\right|} \\
& =\pi \beta^{2}+\beta^{2} h \int_{\theta_{1}}^{\theta_{2}} \frac{d \theta}{\left|f^{\prime}\left(e^{i \theta}\right)\right|} .
\end{aligned}
$$

To find $\int_{\theta_{1}}^{\theta_{2}}\left|f^{\prime}\left(e^{i \theta}\right)\right|^{-1} d \theta$, we change variables via $z=\psi_{r}(s)$ to obtain

$$
\int_{\theta_{1}}^{\theta_{2}} \frac{d \theta}{\left|f^{\prime}\left(e^{i \theta}\right)\right|}=\int_{\tau}^{1} \frac{\left|\psi^{\prime}(i t)\right|^{2}}{\left|F^{\prime}(i t)\right|} d t=\frac{2 r\left(1-r^{2}\right)}{\beta} \int_{\tau}^{1} \frac{t\left(1-t^{2}\right) \sqrt{1-\tau^{2} t^{2}}}{\left(t^{2}+r^{2}\right)^{2} \sqrt{t^{2}-\tau^{2}}} d t
$$

Combining all of these calculations we obtain (4.1).

After integration, an explicit formulation for the maximal omitted area $A(h)$ can be expressed as a function of $r$ that is a complicated combination of polynomials, square roots, and logarithms. Although explicit, this form does not give us any computational advantages. In contrast, to prove the monotonicity of the function $h(r)$ defined by (1.8), it is useful to express the integral in (1.8) in terms of elementary functions. The graph of $h(r)$ is shown in Figure 4.

Changing the variables via $t^{2}=\tau^{2} x$, we can rewrite (1.8) as

$$
h=\beta r\left(1-r^{2}\right) \tau \int_{0}^{1} \frac{\left(1-\tau^{2} x\right)\left(1-\tau^{4} x\right)}{\left(r^{2}+\tau^{2} x\right)^{2}} \frac{d x}{\sqrt{(1-x)\left(1-\tau^{4} x\right)}} .
$$


Expanding the rational function in the integrand into partial fractions and then integrating, yields the following explicit representation for $h=h(r)$ as a function of $r$ :

$$
h(r)=\frac{4\left(1-r^{2}\right)\left(P\left(1+r^{2}\right)-\left(1+r^{4}\right)\right)^{1 / 2}\left(1+3 r^{2}+r^{2}\left(P-1+2 r^{2}\right) Q\right)}{\left(1+r^{2}\right)^{2}\left(1+3 r^{2}\right)^{1 / 2}\left(P-1+2 r^{2}\right)^{1 / 2}\left(r^{2}(P+1)+1-r^{4}\right)^{1 / 2}}
$$

where

$$
P=\left(2-2 r^{2}+r^{4}\right)^{1 / 2} \quad \text { and } \quad Q=\log \frac{P\left(1+r^{2}\right)+r^{2}\left(3-r^{2}\right)}{\left(1+r^{2}\right)\left(2-P+r^{2}\right)} .
$$

Lemma 4.2 The function $h=h(r)$ defined by (4.3) strictly decreases from 4 to 0 as $r$ runs from 0 to 1 .

Proof. Differentiating (4.3), we find

$$
h^{\prime}(r)=\frac{-16 r\left(1-r^{2}\right)\left(\left(c_{0}+c_{1} P\right)+\left(d_{0}+d_{1} P\right) Q\right)}{D},
$$

where

$$
\begin{aligned}
c_{0} & =-96 r^{22}+184 r^{20}+144 r^{18}-318 r^{16}-228 r^{14}+220 r^{12}+296 r^{10} \\
& +868 r^{8}-436 r^{6}+84 r^{4}+64 r^{2}-14, \\
c_{1} & =96 r^{20}-88 r^{18}-280 r^{16} \\
& +34 r^{14}+410 r^{12}+334 r^{10}-50 r^{8}+398 r^{6}-58 r^{4}-38 r^{2}+10, \\
d_{0} & =-32 r^{24}+72 r^{22}+16 r^{20}-124 r^{18}-11 r^{16}+127 r^{14}+1703 r^{12} \\
& -3889 r^{10}+4041 r^{8}-1475 r^{6}-347 r^{4}+361 r^{2}-58, \\
d_{1} & =32 r^{22}-40 r^{20}-72 r^{18}+56 r^{16}+111 r^{14}+3 r^{12} \\
& +1535 r^{10}-2293 r^{8}+1125 r^{6}+121 r^{4}-235 r^{2}+41,
\end{aligned}
$$

and

$$
\begin{aligned}
D & =\left(1+r^{2}\right)^{3}\left(1+3 r^{2}\right)^{3 / 2} P\left(2-P+r^{2}\right)\left(P\left(1+r^{2}\right)+r^{2}\left(3-r^{2}\right)\right) \\
& \times\left(P-1+2 r^{2}\right)^{5 / 2}\left(P\left(1+r^{2}\right)-1-r^{4}\right)^{1 / 2}\left(r^{2}\left(P+1-r^{2}\right)+1\right)^{3 / 2} .
\end{aligned}
$$

It is easily seen that $D$ is non-negative. Hence, to show that $h(r)$ decreases monotonically, it suffices to show that $g=g(r):=\left(c_{0}+c_{1} P\right)+\left(d_{0}+d_{1} P\right) Q$ is non-negative for $0<r<1$.

We will show in Lemma 4.4 below that $0<Q<1$ for $0<r<1$. Hence, to show that $g(r)$ is non-negative on $0<r<1$, it will suffice, in view of the linearity of $g$ in $Q$, to show that

$$
g_{0}=\left(c_{0}+c_{1} P\right)+\left(d_{0}+d_{1} P\right) \cdot 0 \quad \text { and } \quad g_{1}=\left(c_{0}+c_{1} P\right)+\left(d_{0}+d_{1} P\right) \cdot 1
$$

are non-negative for $0<r<1$. By Lemma 4.3 below, we have $s<P<t$, where

$$
s=\frac{19}{50} r^{3}-\frac{9}{10} r^{2}+\frac{1}{125} r+\frac{2827}{2000} \quad \text { and } \quad t=\frac{9}{25} r^{3}-\frac{39}{50} r^{2}+\frac{1}{100} r+\frac{2829}{2000} .
$$

Hence, since $g_{0}$ and $g_{1}$ are linear in $P$, then we have

$$
\min \left\{c_{0}+c_{1} s, c_{0}+c_{1} t\right\} \leq g_{0} \leq \max \left\{c_{0}+c_{1} s, c_{0}+c_{1} t\right\}
$$

and

$$
\min \left\{c_{0}+d_{0}+\left(c_{1}+d_{1}\right) s, c_{0}+d_{0}+\left(c_{1}+d_{1}\right) t\right\} \leq g_{1} \leq \max \left\{c_{0}+d_{0}+\left(c_{1}+d_{1}\right) s, c_{0}+d_{0}+\left(c_{1}+d_{1}\right) t\right\}
$$

Since all the comparison expressions in these formulas are polynomials in $r$ with rational coefficients, we can apply a Sturm sequence argument, see Chapter 5 of [15]. This easily implies that $c_{0}+c_{1} s, c_{0}+c_{1} t, c_{0}+d_{0}+\left(c_{1}+d_{1}\right) s$, and $c_{0}+d_{0}+\left(c_{1}+d_{1}\right) t$ are all non-negative for $0 \leq r \leq 1$. The proof is complete. 

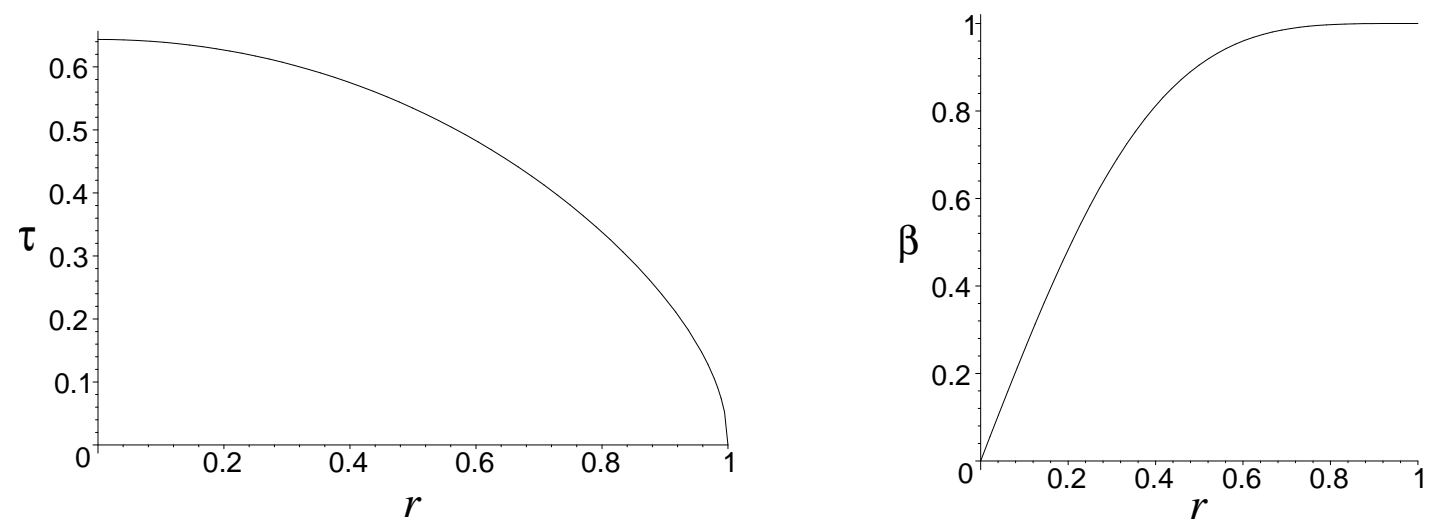

Fig. 5 Functions $\tau(r)$ and $\beta(r)$.

Lemma 4.3 Let $P$ be defined by (4.4) and let $s$ and $t$ be defined by (4.5). Then, $s<P<t$ for $0<r<1$.

Proof. It suffices to show that $s^{2}<P^{2}<t^{2}$ for $0<r<1$. We have

$$
P^{2}-s^{2}=r^{4}-2 r^{2}+2-\left(\frac{19}{50} r^{3}-\frac{9}{10} r^{2}+\frac{1}{125} r+\frac{2827}{2000}\right)^{2}
$$

and

$$
t^{2}-P^{2}=\left(\frac{9}{25} r^{3}-\frac{39}{50} r^{2}+\frac{1}{100} r+\frac{2829}{2000}\right)^{2}-r^{4}+2 r^{2}-2 .
$$

Using a Sturm sequence argument, we can easily see that both $P^{2}-s^{2}$ and $t^{2}-P^{2}$ are non-negative for $0<r<1$.

Lemma 4.4 Let $Q$ be defined by (4.4). Then, $0<Q<1$ for $0<r<1$.

Proof. It suffices to show that $0<Q_{1}<e-1$ for $0<r<1$, where $Q_{1}=\exp (Q)-1$. We will show, in fact, that $0<Q_{1}<3 / 2$, which is equivalent to showing that $Q_{1} /\left(3 / 2-Q_{1}\right)>0$. We can write

$$
\frac{Q_{1}}{3 / 2-Q_{1}}=\frac{\left(4+4 r^{2}\right) P-\left(4+4 r^{4}\right)}{\left(9 r^{2}+10+7 r^{4}\right)-\left(7+7 r^{2}\right) P} .
$$

It is easily seen from (4.4) that $1<P<\sqrt{2}$ for $0<r<1$. Hence, it is clear that the numerator in (4.6) is positive. On the other hand, we have

$$
\left(9 r^{2}+10+7 r^{4}\right)^{2}-\left(7+7 r^{2}\right)^{2} P^{2}=270 r^{4}+82 r^{2}+126 r^{6}+2>0,
$$

which shows that the denominator in (4.6) is positive as well. The lemma is proved.

All the results established so far were used to prove Theorem 1.1. Now we prove monotonicity properties of the functions $\tau=\tau(r), \beta=\beta(r)$, and $a=a(r)$. Although not needed for our main proof they provide some additional information about extremal configurations. The graph of $a(r)$ is displayed in Figure 4 and the graphs of functions $\tau(r)$ and $\beta(r)$ are displayed in Figure 5. 1.

Lemma 4.5 The function $\tau=\tau(r)$ defined by (1.9) strictly decreases from $\sqrt{\sqrt{2}-1}$ to 0 as $r$ runs from 0 to 
Proof. It suffices to work with $\tau^{2}=\tau^{2}(r)$. Differentiating $\tau^{2}$ we obtain

$$
\frac{d \tau^{2}}{d r}=\frac{2 r}{P\left(1+3 r^{2}\right)^{2}} p(r),
$$

where $P$ is defined by (4.4) and

$$
p(r)=-5+r^{2}-r^{4}+3 r^{6}+\left(3-2 r^{2}-3 r^{4}\right) P .
$$

Hence, it suffices to show that $p(r)$ is negative for $0 \leq r \leq 1$. It is easily seen that $P$ decreases from $\sqrt{2}$ to 1 as $r$ varies from 0 to 1 . Hence, for $0 \leq r \leq 1$, we have $1 \leq P<3 / 2$. Suppose that

$$
\begin{aligned}
& c_{1}(r)=-5+r^{2}-r^{4}+3 r^{6}+\left(3-2 r^{2}-3 r^{4}\right) \cdot 1, \\
& c_{2}(r)=-5+r^{2}-r^{4}+3 r^{6}+\left(3-2 r^{2}-3 r^{4}\right) \cdot(3 / 2) .
\end{aligned}
$$

The linearity of $p$ with respect to $P$ implies that

$$
\min \left\{c_{1}(r), c_{2}(r)\right\} \leq p(r) \leq \max \left\{c_{1}(r), c_{2}(r)\right\}
$$

Using a Sturm sequence argument, it is easily seen that both $c_{1}(r)$ and $c_{2}(r)$ are negative for $0 \leq r \leq 1$. Thus, $\tau^{2}(r)$ decreases on $0 \leq r \leq 1$ and the lemma follows.

Lemma 4.6 Let $\beta=\beta(r)$ be defined by (1.10) with $\tau=\tau(r)$ defined by (1.9). Then, $\beta$ strictly increases from 0 to 1 as $r$ runs from 0 to 1 .

Proof. It suffices to show that $\beta^{2}$ is an increasing function of $r$, which maps $[0,1]$ onto $[0,1]$. We obtain, after some algebra,

$$
\beta^{2}=\frac{16 r^{2}\left(2 r^{4}+r^{2}-1\right)+16 r^{2}\left(1+r^{2}\right) P}{\left(1+r^{2}\right)^{4}\left(1+2 r^{2}-r^{6}\right)+\left(1+r^{2}\right)^{4}\left(r^{4}+r^{2}\right) P},
$$

where $P$ is defined by (4.4). Differentiating $\beta^{2}$, we find

$$
\frac{d \beta^{2}}{d r}=\frac{32 r\left(1-r^{2}\right)^{2}\left(1+r^{2}\right)^{5}}{P\left(\left(1+r^{2}\right)^{4}\left(1+2 r^{2}-r^{6}\right)+\left(1+r^{2}\right)^{4}\left(r^{4}+r^{2}\right) P\right)^{2}} p(r),
$$

where

$$
p(r)=-4 r^{6}-r^{4}-5 r^{2}+2+\left(4 r^{4}+5 r^{2}-1\right) P .
$$

Hence, it suffices to show that $p(r)$ is non-negative for $0 \leq r \leq 1$. Now using a Sturm sequence argument, one can finish the proof as in the previous lemma.

Since $r=r(h)$ is monotonic on $0<h<4$, the parameters $\tau$ and $\beta$ in the definition of the extremal function $f_{h}$ of Theorem 1.1 are monotonic functions of $h$. It is worth mentioning that the third natural parameter, $a=a(h)$, which gives the length of the vertical segment of the non-free boundary, is not monotonic in $h$. It is easy to see that the disk $\{w:|w-1| \leq 1\}$ and segment $[0,4]$ are the limit extremal configurations for the problem under consideration. Thus, $a=0$ in both limit cases. Our next lemma shows however that $a=a(r)$ considered as a function of $r$ has only one local maximum on $0<r<1$.

Lemma 4.7 Let $a=a(r)$ be defined by (4.2) with $\tau$ and $\beta$ defined by (1.9) and (1.10). Then, there is a unique $r_{1}, 0<r_{1}<1$, such that a $(r)$ strictly increases as $r$ varies from 0 to $r_{1}$ and strictly decreases as $r$ varies from $r_{1}$ to 1 .

Proof. Upon integration, $a(r)$ can be expressed as an explicit function of $r$ which is a combination of polynomials, square roots, and arctangents. We give here an argument that is reminiscent of the argument given in the proof of Lemma 4.2, omitting some of the technical details. For convenience, we set $r_{0}=53 / 100$ and $r_{2}=57 / 100$. 
Differentiating $a(r)$ with respect to $r$ we obtain a representation

$$
a^{\prime}(r)=4 r\left(1-r^{2}\right) \frac{\left(c_{0}+c_{1} P\right)+\left(d_{0}+d_{1} P\right) G(r)}{D_{1}(r)}
$$

where $P=\left(2-2 r^{2}+r^{4}\right)^{1 / 2}$, the functions $G$ and $D_{1}$ are non-negative on $(0,1)$ and $c_{0}, c_{1}, d_{0}$ and $d_{1}$ are polynomials in $r$ with rational coefficients. We will show that there exists an $r_{1}$ such that $a^{\prime}(r)>0$ on $\left(0, r_{1}\right)$ and $a^{\prime}(r)<0$ on $\left(r_{1}, 1\right)$.

Using the linearity of the terms $c_{0}+c_{1} P$ and $d_{0}+d_{1} P$ in $P$ and the estimates on $P$ given in Lemma 4.3, one can give a Sturm sequence argument to show that $c_{0}+c_{1} P>0$ and $d_{0}+d_{1} P>0$ on the interval $\left(0, r_{0}\right)$ and that $c_{0}+c_{1} P<0$ and $d_{0}+d_{1} P<0$ on the interval $\left(r_{2}, 1\right)$. Hence, $a^{\prime}(r)>0$ on $\left(0, r_{0}\right)$ and $a^{\prime}(r)<0$ on $\left(r_{2}, 1\right)$.

We define $n(r)=\left(c_{0}+c_{1} P\right)+\left(d_{0}+d_{1} P\right) G(r)$. Differentiating $n(r)$ with respect to $r$ we obtain a representation

$$
n^{\prime}(r)=2 r \tau^{2} \frac{\left(\tilde{c}_{0}+\tilde{c}_{1} P\right)+\left(\tilde{d}_{0}+\tilde{d}_{1} P\right) G(r)}{D_{2}(r)}
$$

where the function $D_{2}$ is non-negative on $(0,1), \tau$ is defined by (1.9) and $\tilde{c}_{0}, \tilde{c}_{1}, \tilde{d}_{0}$ and $\tilde{d}_{1}$ are polynomials in $r$ with rational coefficients.

Using the linearity of terms $\tilde{c}_{0}+\tilde{c}_{1} P$ and $\tilde{d}_{0}+\tilde{d}_{1} P$ in $P$ and the estimates on $P$ given in Lemma 4.3, one can give a Sturm sequence argument to show that $\tilde{c}_{0}+\tilde{c}_{1} P<0$ and $\tilde{d}_{0}+\tilde{d}_{1} P<0$ on the interval $\left(r_{0}, r_{2}\right)$ and, hence, that $n(r)$ is strictly decreasing on the interval $\left(r_{0}, r_{2}\right)$ and changes sign exactly once. Consequently, $a^{\prime}(r)$ changes sign exactly once on $\left(r_{0}, r_{2}\right)$.

The value $r_{1}$ is the unique solution of $n(r)=0$, which lies in the interval $\left(r_{0}, r_{2}\right)$.

\section{Some remarks and problems}

(a) Omitted area problem. The following problem proposed by A. W. Goodman [13] can be considered as a prototype of all omitted area problems with geometrical constraints: Find $A:=\inf _{f \in S}\{\operatorname{Area}(f(\mathbb{D}) \cap \mathbb{D})\}$ over the standard class $S$ of univalent functions $f$ in $\mathbb{D}$ with $f(0)=0, f^{\prime}(0)=1$.

To our knowledge, this problem remains open although many important properties of extremal functions have been proved since 1949. Here we summarize some of them. If $f \in S, f$ is extremal for $A$ and $f(1)=\infty$, then $D=f(\mathbb{D})$ is circularly symmetric w.r.t. $\mathbb{R}_{0}$ and there exist $\theta_{1}, \theta_{2}$, and $\beta$ such that $0<\theta_{1}<\theta_{2}<\pi, 0<\beta<1$, and $f$ satisfies the following boundary conditions:

(a) $\Im f\left(e^{i \theta}\right)=0$ for $0<|\theta| \leq \theta_{1}$;

(b) $\left|f\left(e^{i \theta}\right)\right|=1$ for $\theta_{1}<|\theta|<\theta_{2}$;

(c) $\left|f^{\prime}\left(e^{i \theta}\right)\right|=\beta$ for $\theta_{2}<\theta<2 \pi-\theta_{2}$;

(d) $f^{\prime}$ has a non-zero continuous extension to $\mathbb{D} \cup\left\{e^{i \theta}: \theta_{1}<\theta<2 \pi-\theta_{1}\right\}$ which is Hölder-continuous with exponent $1 / 2$;

(e) $\left|f^{\prime}\left(e^{i \theta}\right)\right|$ strictly decreases in $\theta_{1}<\theta<\theta_{2}$;

(f) there is a $\theta_{0}, 0<\theta_{0}<\theta_{1}$ such that $\left|f^{\prime}\left(e^{i \theta}\right)\right|$ strictly decreases from $+\infty$ to $\beta_{1}$, where $\beta_{1}>\beta$, and strictly increases from $\beta_{1}$ to $+\infty$ in $0<\theta<\theta_{0}$ and $\theta_{0}<\theta<\theta_{1}$, respectively.

Observations (a) and (b) were made by Barnard and Suffridge, see [10, p. 536]. Condition (d) was proved by J. Lewis [16] who also proved that (c) holds true for all $\theta$ except the set $I=\left\{e^{i \theta}: \Im f\left(e^{i \theta}\right)=0\right\}$ which may consists of at most a finite number of closed arcs. The inequality $\beta<1$ and conditions (e), (f), and (c) without the above mentioned exception were established in [9]. 
The conclusion of Lemma 2.2(d) that the vertical non-free boundary is not degenerate, i.e., that there is a strict inequality $\theta_{1}<\theta_{2}$ for the parameters $\theta_{1}$ and $\theta_{2}$ of this iceberg-type problem is reminscient of the conclusion in [16] that there is a strict inequality $\theta_{1}<\theta_{2}$ for the parameters $\theta_{1}$ and $\theta_{2}$ of Goodman's omitted area problem. With minor modifications, the proof in [16] that $\theta_{1}<\theta_{2}$ for Goodman's omitted area problem could have been modified to prove Lemma 2.2(d). In this paper, we have given an independent proof of Lemma 2.2(d) and we mention here that, alternatively, with minor modifications the proof of Lemma 2.2(d) could be used to show that $\theta_{1}<\theta_{2}$ for Goodman's omitted area problem as well. Approximations to the exact value of $A$ have been given in $[3,5]$ by different numerical methods. In particular, [3] suggests that $A=0.2385813284 \pi$, where all explicitly shown digits are exact.

(b) Width of the invisible part of the iceberg. The method of this paper can be also applied to find the extremal function for Problem (1.4)(b) if one can show a priori that the free boundary of the extremal is smooth enough. One difference compared to Problem (1.4)(a) is that the extremal configurations now do not possess circular symmetry although they still possess Steiner symmetry. In view of this lack of symmetry, we cannot apply the local variations developed in Section 2 since the boundary may be non-rectifiable. Perhaps, the necessary smoothness can be achieved by applying a more powerful technique such as that of J. Lewis [16] mentioned in the Introduction.

(c) Safe distance from the iceberg. The situation with Problem (1.4)(c) differs from the other two cases. To explain this, we start with the limiting case when the whole iceberg is observable, i.e. when area $\left(E_{+}\right)=\pi$. Then, of course, $E$ coincides with the disk $\{w:|w-(1+i)| \leq 1\}$ up to translation along the real axis.

This disk has a contact point with the surface of interface at $z=1$ and a contact point with the front line, which coincides with the imaginary axis, at $z=i$. These two contact points represent the non-free boundary in this limiting case. It is reasonable to expect that for icebergs with visible area slightly less than $\pi$, the extremal configurations will have two disjoint segments, vertical and horizontal, as their non-free boundary. If so, then transplanting the problem into the auxiliary $s$-plane as in Section 3, we have to deal with the omitted area problem for functions defined in a doubly-connected domain. To our knowledge, there are no known solutions of problems of this kind.

(d) Convex icebergs. Let $\mathcal{F}^{c}$ denote the collection of all convex compact sets $E$ in $\mathcal{F}$. It will be interesting to study problems (1.4) for the class $\mathcal{F}^{c}$. Since there are more available methods for convex sets and functions, there is a chance that known techniques may give complete solutions to all three problems.

\section{References}

[1] D. Aharonov, H. S. Shapiro, and A. Yu. Solynin, A minimal area problem in conformal mapping. J. Analyse Math. 78 (1999), 157-176.

[2] D. Aharonov, H. S. Shapiro, and A. Yu. Solynin, Minimal area problems for functions with integral representation. J. Analyse Math. 98 (2006), 83-111.

[3] L. Banjai, L. Trefethen, Numerical solution of the omitted area problem of univalent function theory. Comp. Methods Func. Theory 1 (2001), no. 1, 259-273.

[4] R. W. Barnard, J. L. Lewis, On the omitted area problem. Michigan Math. J. 34 (1987), 13-22.

[5] R. W. Barnard, K. Pearce, Rounding Corners of Gearlike Domains and the Omitted Area Problem. J. Comp. Appl. Math. 14 (1986), 217-226; Numerical Conformal Mapping, edited by L. N. Trefethen. North Holland, 1986.

[6] R. W. Barnard, K. Pearce, and A. Yu. Solynin, An isoperimetric inequality for logarithmic capacity. Annales Academiæ Scientiarum Fennicæ. Mathematica 27 (2002), 419-436.

[7] R. W. Barnard, C. Richardson, and A. Yu. Solynin, Concentration of area in half-planes. Proc. Amer. Math. Soc. 133 (2005), no. 7, 2091-2099.

[8] R. W. Barnard, C. Richardson, and A. Yu. Solynin, A minimal area problem for nonvanishing functions. Algebra i Analiz 18 (2006), no. 1, 35-54; English translation in: St. Petersburg Math. J. 18 (2007), no. 1, 21-36.

[9] R. W. Barnard and A. Yu. Solynin, Local variations and minimal area problem for Carathéodory functions. Indiana U. Math. J. 53 (2004), no. 1, 135-167.

[10] D. Brannan and J. Clunie, Aspects of Contemporary Complex Analysis. Academic Press, New York, 1980. 
[11] V. N. Dubinin, Symmetrization in geometric theory of functions of a complex variable. Uspehi Mat. Nauk 49 (1994), 3-76 (in Russian); English translation in: Russian Math. Surveys 49: 1 (1994), 1-79.

[12] P. Duren, Univalent Functions. Springer-Verlag, 1992.

[13] A. Goodman, Note on regions omitted by univalent functions. Bull. Amer. Math. Soc. 55 (1949), 363-369.

[14] W. K. Hayman, Multivalent Functions. Second Edition. Cambridge Tracts in Mathematics, 110. Cambridge Univ. Press, Cambridge, 1994.

[15] N. Jacobson, Basic Algebra. I. Second Edition. W. H. Freeman and Company, New York, 1985.

[16] J. L. Lewis, On the minimal area problem. Indiana Univ. Math. J. 34 (1985), 631-661.

[17] Ch. Pommerenke, Boundary Behaviour of Conformal Maps. Springer-Verlag, 1992. ath. J. 8 (1997), 1015-1038.

[18] A. Yu. Solynin, Functional inequalities via polarization. Algebra i Analiz 8 (1996), 145-185; English translation in: St. Petersburg Math. J. 8 (1997), 1015-1038. 